1 Jotirao Phule

(1827-1890), E.V.

Ramaswamy Nayker (popularly known as Periyar (1879-1973), Bhimrao Ambedkar (1891-1956).
Attempts to trace the origins of exploitation and oppression are extremely relevant. However, it is also important for both the colonized and the colonizers to locate the multiplicities of oppression from within and beyond and from the nation to the Empire. And so, when we in India try to trace alternative democracies in our past, we not only read Gandhi (pp. 101-104), Tagore (pp. 106-108) or Sri Aurobindo (pp. 108-110), but also try to understand thinkers like Phule, Periyar or Ambedkar ${ }^{1}$ for their contributions towards making India meaningfully democratic.

In India, religious politics have done more harm than good to its unity. And that is why whenever Indian nationalism's imagined community stood for 'unity in diversity', India's poor, women, tribals and dalits fought for diversity - whether in Tamil Nadu, North East or Kashmir. For the white people in the West and for the upper caste in the East, a major contribution by the anti-racist/anti-casteist feminist politics will be, as Eisenstein illustrates, a humanitarianism that is polyversal. Quoting Toni Morrison, Eisenstein points out, 'Definitions belong to the definers, not the defined' ( $p .188$ ). The attempts by the West to define the Black or the Muslim belonged to the Westerners; it doesn't belong to the defined. And equally problematic has been the attempts of the East (living in the West or the non-West) to define the dalit or the subaltern.

If the polyversal world, as Eisenstein explains, wishes to have a 'view from everywhere', then feminists across the world will have to see and think more on many other fundamentalisms beyond the Muslim. Or to be more precise, Western feminists will have to tell the West to open their eyes towards Christian fundamentalism. Yet, how do third-world feminists confront the Empire? Eisenstein shows a very rarely visible passion and honesty to say that it is important for feminisms today more than ever to think polyversally and to see human bodies beyond colour and language. Yet, I was left wanting more specificity about exactly what such a polyversal account would entail and for whom. What her book contributes to is the hope that polyversal feminisms of the West and the East will see beyond boundaries created between empires and nations and the definers and the defined by capital and power.

M.S. Sreerekha

doi:10.1057/palgrave.fr. 9400295

\section{Feeding anorexia: gender and power at a treatment center}

Helen Gremillion; Duke University Press, Durham and London, 2003, 280p, ISBN 0-8223-3133-0 (Hbk) £69.00; ISBN 0-8223-3120-9 (Pbk) £16.95

Feeding Anorexia is a refreshingly original and insightful account of how anorexia is (re)constituted. Through careful ethnographic investigation and astute critical 
analysis, Gremillion shows how the regime of a leading treatment centre for eating disorder sufferers in the west of the USA regenerates the very power relations that it identifies as part of anorexia's conditions of emergence. Gremillion's analysis marks a welcome shift away from much feminist social constructionist and poststructuralist theory about anorexia of the 1990s that tended to begin from a generalized model for understanding how power acts on (female) bodies, then apply that model to anorexia. By documenting how power relations are negotiated and regenerated in a specific context, Gremillion presents a sophisticated account of the effects of dominant discourses about anorexia and a sensitive description of how anorexia is experienced by sufferers and their parents. Feeding Anorexia is, as its author suggests, important 'groundwork for advocacy' (p. 23) and has far-reaching political implications. It is just what feminist literature on anorexia has been waiting for.

Drawing richly on her ethnographic material, Gremillion unsettles a core presumption of much feminist literature on anorexia: that psychiatric and 'cultural' explanations for anorexia are historiographically and epistemologically opposed. Indeed, not only are pathologized and socio-cultural discourses mutually constitutive, she argues, but it is precisely through psychiatric claims to objectivity that dominant 'cultural' ideas about individual and familial health and identity are thrown into relief (p. 32). As well as undermining the perceived binary relationship between 'nature' and 'culture' in arguments about anorexia's aetiology, Gremillion helpfully disrupts the frequent presumption that agency is discursively opposed to docility, or subversion to conformity. Through detailed descriptions of the relationships, practices, protocols, and decision-making procedures in the treatment centre, she demonstrates the ways in which apparent acts of resistance on the part of anorexic patients often have conservative effects, and seeming acts of compliance can challenge the prevailing order by exposing contradiction and conflict in the treatment programme. For example, she shows that for patients diagnosed with 'typical' anorexia, acts of direct resistance, such as in expressions of willfulness or anger, become enfolded back into dominant clinical interpretations of anorexia and are read positively as evidence of a nascent sense of identity deemed to be lacking in anorexics. Conversely, it is through exaggeration or hyperbolic compliance with social and clinical norms relating to self-control and fitness, she notes, that anorexic patients uncover instabilities in the ideology and practices of treatment and, consequently, highlight the gendered character and effects of discourses about the 'healthy' body.

The argument that contradictory or conflicting discourses collide in anorexia is not unusual in recent feminist theory about anorexia. But what makes Gremillion's analysis different is her attention to the actual, situated effects of such contradictions. In Chapter 1 , she details how the treatment programme's precise management of patients' calorific intake and exercise accentuates the 
gendered cultural imperative that sees the body as a resource for selfactualization and the female body in particular as an encumbrance in this project. Because decisions about treatment are presented to patients as objective criteria for health, with conflict or disagreement among practitioners concealed, patients experience their own resistance as evidence of pathology. In this way, Gremillion argues, treatment practices generate precisely the senses of dependency and lack of identity that they set out to correct.

Continuing her analysis of the effects of treatment, Gremillion turns her attention, in Chapter 2, to structural family therapy. She details how a contemporary ideology of 'minimal mothering', infusing the treatment centre's approach, leaves mothers of anorexic patients in an impossible position in which they are both invited to blame themselves for being too 'overbearing' and yet, on their daughter's discharge, expected instinctively to supply the right level of love and support for continued recovery. Mothers are expected to withdraw from the treatment process, to facilitate separation and individuation, and yet exclusion generates the very desire for interdependency it seeks to redress. Further ironies abound in relation to the treatment team's conception of itself as a 'substitute family', which Gremillion explores in Chapter 3. The hierarchical gendered structure of the 'therapeutic' family reproduces many of the conditions identified as constitutive of anorexia in patients' 'natural' families. For example, female caretakers tend to monitor themselves critically for signs of 'over-involvement' with patients, while in practice a close attentiveness to patients is often generated in response to harsh treatment interventions on the part of male psychiatrists. In the process, these substitute fathers, like patients' 'natural' fathers, are absolved from taking responsibility for any part they might play in their daughters'/patients' distress.

Gremillion's analysis, in Chapter 4, of the ways in which dominant categories of individual and familial health and illness are constituted through ideologies of 'race' and class, is rare indeed in feminist literature on anorexia. She reveals how the profile of the 'typical' anorexic as white and middle class is regenerated, in circular fashion, through the marginalization and exclusion of 'borderline' patients who are often working class and/or black and who are cast as beyond the help of treatment because of their alleged inability to strike a 'healthy' balance between compliance and resistance. In their guise as objective descriptions, diagnostic categories like 'borderline' thus work to neutralize the racist and classist ideologies that structure them such that the responsibility for their effects is carried by the 'borderline' patients themselves.

Feeding anorexia closes with an epilogue which points to narrative therapy as an alternative set of possibilities for treating sufferers of anorexia, and one which is compatible with Gremillion's analysis of power. While this section provides a closing note of optimism, I wondered whether Gremillion's enthusiasm for narrative therapy left her, at times, slightly more forgiving of its inconsistencies 
than she had been for more mainstream treatment practices. For example, the use of language like 'anti-anorexic life story' (p. 207) seems in tension with Gremillion's argument that narrative therapy does not necessarily render anorexia 'separate' from the person suffering it or 'bad'. However, such tensions are not altogether out of place in the speculative space of an epilogue and are no doubt already proving constructive in Gremillion's current research on therapies informed by poststructuralist ideas about gender and power.

doi:10.1057/palgrave.fr. 9400296

Jo Brain 